\title{
Diversidad de la herpetofauna en la Reserva Natural Datanlí - El Diablo, Santa Maura (Jinotega)
}

Heraldo Ramón Salgado Aráuz ${ }^{1}$ y Francisco José Blanco ${ }^{2}$

Profesor investigador de la Facultad de Ciencia, Tecnología y Ambiente, Universidad Centroamericana, Apdo. 69. Managua, Nicaragua. E-mail: Sheraldo_ramon@yahoo.com

2 Estudiante de Ingeniería en Calidad Ambiental, Asistente de investigación, Facultad de Ciencia, Tecnología y Ambiente, Universidad Centroamericana.

ESTA INVESTIGACIÓN SE REALIZÓ En LA RESERVA NATURAL Datanlí - El Diablo, ubicada a 27 Km de la ciudad de Jinotega, al Noroeste del mismo departamento. Se realizaron muestreos por parcelas de 10 x $10 \mathrm{~m}$ y transectos irregulares de $3 \mathrm{Km}$ cada uno de forma determinante de acuerdo con los tipos de microhábitats que prefieren los anfibios y reptiles. Se determinó una composición de 66 especies, 42 géneros y 14 familias. La composición para ambos taxones fue la siguiente: 35 especies, 27 géneros y 7 familias que pertenecen a los reptiles; 31 especies, 15 géneros y 7 familias correspondientes a los anfibios. El total de especies de la herpetofauna encontrada se obtuvo a partir de un registro total de 373 individuos. Las familias más representativas fueron para reptiles: Colubridae con 18 especies y 102 individuos, Iguanidae con 8 especies y 70 individuos y Viperidae con 5 especies y 21 individuos. Para anfibios fueron: Leptodactylidae con 10 especies y 58 individuos, Hylidae con 10 especies y 51 individuos y Centrolenidae con 4 especies y 10 individuos. Los 4 microhábitats fueron heterogéneos por presentar especies asociadas a ellos.

Palabras clave: anfibios-distribución geográfica (Nicaragua) / reptilesinvestigaciones

\section{Introducción}

Por su ubicación geográfica y configuración climática, Nicaragua cuenta con gran diversidad de especies silvestres en sus ecosistemas, lo que la hace ser muy rica debido a la variedad de hábitats que éstos ofrecen. A pesar de esto, no se cuenta aún con suficientes estudios biológicos que brinden la información necesaria sobre su diversidad faunística, por lo que su exploración se encuentra todavía muy incipiente.

La Reserva Natural Datanlí - El Diablo cuenta con una extensión de 5.848 hectáreas. Ésta comprende cañadas de gran importancia hidrológica que nacen en la parte de arriba del cerro, entre las que se destaca el río Santa Maura con un caudal abundante aún en época de verano y con una caída total de unos $200 \mathrm{~m}$ hasta el valle. En el área se despliegan condiciones 
topográficas que incluyen pendientes de hasta un 65\% con un relieve muy quebrado. Es un área de gran importancia e interés nacional sobre todo por sus manifestaciones tropicales debido a la configuración climática de la zona.

El presente estudio tiene como propósito recopilar información básica sobre la diversidad de la herpetofauna (reptiles y anfibios) por microhábitats de Datanlí - El Diablo, que está aún sin caracterizar. Pretende proporcionar bases más amplias para su aprovechamiento en el área o para la ejecución de los planes de manejo de la misma, y a la vez contribuir al mantenimiento y/o preservación de las especies.

\section{Metodología}

Área de estudio:

La Reserva Natural Datanlí - El Diablo se encuentra ubicada a 27Km al Noroeste de la ciudad de Jinotega en las elevaciones geológicas más antiguas del país, arriba de los 1600 msnm. Por lo general se encuentra cubierta por neblina. Presenta un clima subtropical en sus cumbres más altas, con una temperatura promedio anual de $20^{\circ} \mathrm{C}$ y una precipitación pluvial que oscila entre los 1600 y $2000 \mathrm{~mm}^{3}$ durante los meses más lluviosos (Incer, 1998:9-45).

\section{Categorías de microhábitats:}

Dentro del área de estudio se definieron cuatro tipos de microhábitats, considerados como unidades naturales de muestreo, en los que se determinó la presencia de anfibios y reptiles. Los tipos de microhábitats son los siguientes: suelo/hojarasca, fuste, follaje/ramas y riachuelos/rocas. Éstos fueron definidos de acuerdo a condiciones ambientales observadas in situ en el área, así como por los hábitos y preferencias de hábitats que estas especies requieren para establecerse.

\section{Métodos de muestreo:}

En el "muestreo por parcelas" se estableció un total de 100 parcelas de 10 x 10 m, que fueron situadas de forma selectiva de acuerdo al tipo de microhábitat en el área de estudio.

El "muestreo por transectos irregulares" se estableció de forma selectiva de acuerdo al microhábitat con un tamaño de $3 \mathrm{Km}$ cada uno, realizando un total de 20 transectos en el área (Heyer et al., 1994:97-102).

\section{Muestreo en parcelas y transectos:}

Se utilizó un GPS durante el reconocimiento de campo para determinar la localización y el establecimiento de ambos métodos de muestreo en el área tomando en cuenta el tipo de microhábitat considerado.

Se efectuó una búsqueda intensiva en cada parcela y transecto establecido haciendo uso de una guía de observación de campo que brindó un listado de las especies encontradas de acuerdo a su abundancia relativa (Heyer et al., 1994:97-102). 
La actividad de muestreo se realizó en noviembre de 2005, enero y mayo de 2006, en horas de mayor actividad de reptiles y anfibios. En el caso de los primeros, son animales diurnos, crepusculares y nocturnos; en el caso de los segundos sus hábitos son nocturnos y crepusculares, sólo unos cuantos son eminentemente diurnos. Por lo tanto, el horario de actividad de muestreo fue de 8:00 AM a 12:00 AM por la mañana y de 4: 00 PM a 10:00 PM por la tarde y noche.

\section{Técnicas de captura:}

El trampeo se realizó diariamente. Se utilizaron las siguientes trampas:

a) Trampas de embudo (Ilustración 1), instalándose 12 en total, siendo distribuidas al azar y rotadas una en cada parcela hasta cubrir las 100 parcelas de muestreo con un esfuerzo diario de aproximadamente seis parcelas;

b) Captura de anfibios y reptiles a mano limpia; y

c) Captura de reptiles utilizando una caña de madera (Ilustración 2).

Los 20 transectos se recorrieron solamente una vez, y sólo se tomaba en cuenta los encuentros visuales y casuales con las especies.

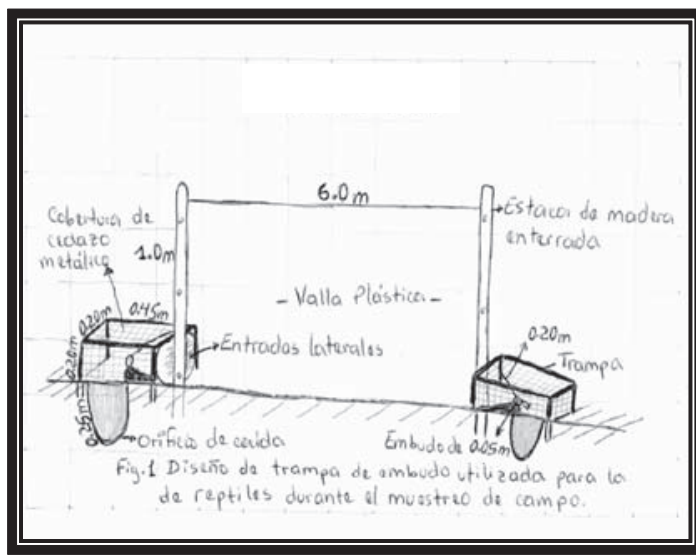

Ilustración 1. Trampas de embudo
CONTINUACION DE ANEXONOB

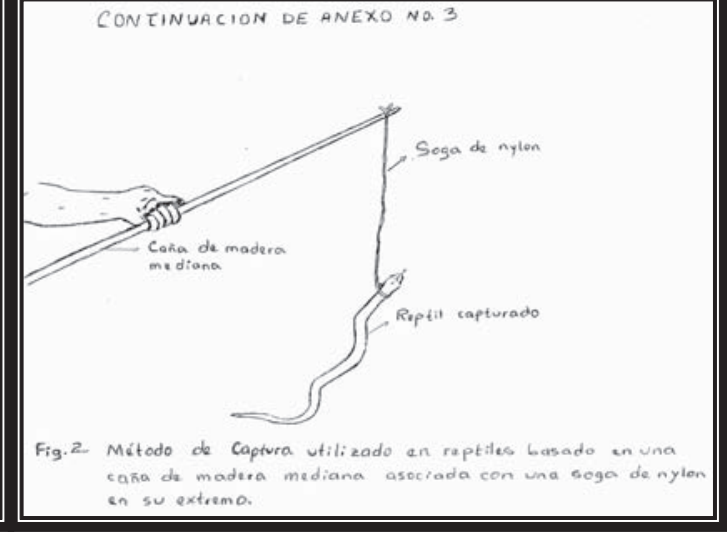

Ilustración 2. Captura con caña de madera

\section{Determinación taxonómica:}

Las especies observadas en el campo y capturadas fueron identificadas a través de comparaciones con fotografías de las especies contenidas en Ruíz (1996:7-10), Ruiz y Buitrago (2003:337), Kölher (1998:1-5; 2001:207), y comparaciones con especimenes de la colección del Museo de Ciencias Naturales de la Universidad Centroamericana (UCA), contando con el apoyo de especialistas en el campo de la Herpetología (Msc. Gustavo Adolfo Ruíz) para comprobar la identificación de las especies. Las especies colectadas fueron depositadas en el área para formar parte del Centro de información ambiental. 
Análisis de los resultados:

Se usaron los índices de diversidad de Shannon $\left\{\hat{\mathrm{H}}^{\prime}=-\sum_{\mathrm{i}=1}^{\mathrm{s}^{*}}\left(\mathrm{p}_{1} \ln \mathrm{p}_{\mathrm{f}}\right)\right\}$ y Simpson $\left\{\hat{\lambda}=\sum_{\mathrm{i}=1}^{\mathrm{s}} \frac{\mathrm{n}_{\mathbf{1}}\left(\mathrm{n}_{\mathrm{n}}-\mathbf{1}\right)}{\mathrm{n}(\mathrm{n}-\mathbf{1})}\right\}$ por tipo de microhábitat considerado, así como los índices de equitatividad $\mathrm{E} 1\left\{\mathrm{E} 1=\frac{\mathrm{H}^{\prime}}{\ln (\mathrm{S})}=\frac{\ln (\mathrm{N} 1)}{\ln (\mathrm{N} 0)}\right\}$ y E5 $\left\{\mathrm{E} 5=\frac{(1 / \lambda)-1}{\mathrm{e}^{\mathrm{H}^{\prime}}-1}=\frac{\mathrm{N} 2-1}{\mathrm{~N} 1-1}\right\}$ basados en los números de diversidad de Hill (Ludwig \& Reynolds, 1988:65-103; Krebs, 1989:495-513; Bach, 1998: 12,18,66) para determinar la equitatividad en la distribución de la abundancia de las especies por microhábitat, tomando en cuenta el número de especies (S) de cada microhábitat.

Se aplicó el índice cuantitativo de Sorensen $\left\{C_{N}=\frac{2_{j N}}{(a N+b N)}\right\}$ para el análisis de la heterogeneidad en la composición de especies (Magurran, 1988:94-99).

\section{Resultados}

Composición y diversidad de la herpetofauna:

La herpetofauna, durante el período de estudio, estuvo conformada por 66 especies, 42 géneros y 14 familias, cuya distribución para ambos taxones fue la siguiente: 35 especies, 27 géneros y 7 familias de reptiles; 31 especies, 15 géneros y 7 familias de anfibios. El total de especies de la herpetofauna encontrada fue de 373 individuos (228 de reptiles y 145 de anfibios observados). 
Cuadro 1. Composición y abundancia relativa de reptiles por familias y especies

\begin{tabular}{|c|c|c|c|}
\hline No. & Nombre científico & Abundancia relativa & Familia \\
\hline 1 & Norops humilis & 10 & Iguanidae \\
\hline 2 & Norops cupreus & 3 & Iguanidae \\
\hline 3 & Norops lemurinus & 6 & Iguanidae \\
\hline 4 & Norops capito & 12 & Iguanidae \\
\hline 5 & Norops biporcatus & 6 & Iguanidae \\
\hline 6 & Sceloporus malachiticus & 15 & Iguanidae \\
\hline 7 & Basiliscus plumifrons & 8 & Iguanidae \\
\hline 8 & Basiliscus basiliscus & 10 & Iguanidae \\
\hline 9 & Leptophis ahaetulla & 12 & Colubridae \\
\hline 10 & Imantodes cenchoa & 4 & Colubridae \\
\hline 11 & Drymobius margaritiferu & 2 & Colubridae \\
\hline 12 & Lampropeltis triangulum & 9 & Colubridae \\
\hline 13 & Oxybelis fulgidus & 12 & Colubridae \\
\hline 14 & Ninia maculata & 2 & Colubridae \\
\hline 15 & Pliocercus euryzonus & 13 & Colubridae \\
\hline 16 & Rhadinaea decorata & 5 & Colubridae \\
\hline 17 & Spilotes pullatus & 10 & Colubridae \\
\hline 18 & Stenorrhina freminville & 7 & Colubridae \\
\hline 29 & Drymobius chloroticus & 8 & Colubridae \\
\hline 20 & Dryadophis dorsalis & 2 & Colubridae \\
\hline 21 & Hydromorphus concolor & 1 & Colubridae \\
\hline 22 & Oxyrhopus petola & 7 & Colubridae \\
\hline 23 & Oxybelis brevirostris & 2 & Colubridae \\
\hline 24 & Nothopsis rugosus & 2 & Colubridae \\
\hline 25 & Pseustes poecilonotus & 2 & Colubridae \\
\hline 26 & Sibon annulatus & 2 & Colubridae \\
\hline 27 & Atropoides nummifer & 8 & Viperidae \\
\hline 28 & Lachesis muta & 4 & Viperidae \\
\hline 29 & Agkistrodon bilineatus & 3 & Viperidae \\
\hline 30 & Bothriechis schlegelii & 2 & Viperidae \\
\hline 31 & Porthidium nasutum & 4 & Viperidae \\
\hline 32 & Celestus bivittatus & 2 & Anguidae \\
\hline 33 & Sphenomorphus cherriei & 6 & Scincidae \\
\hline 34 & Micrurus nigrocintus & 20 & Elapidae \\
\hline 35 & Boa constrictor & 7 & Boidae \\
\hline
\end{tabular}

Fuente: Elaboración propia.

En el Cuadro 1 se plasman los datos representativos de los reptiles encontrados. Las familias mejor representadas fueron: Colubridae con 18 especies y 102 individuos, Iguanidae con 8 especies y 70 individuos y Viperidae con 5 especies y 21 individuos. Las familias menos representativas fueron: Elapidae con una especie Micrurus nigrocintus representada por 20 individuos, Scincidae con una especie Sphenomorphus cherriei y 6 individuos y Boidae con una especie Boa constrictor y 7 individuos. 
Las especies más abundantes durante el muestreo fueron: Micrurus nigrocintus con 20 individuos, siendo la especie más representativa del área, seguida de Sceloporus malachiticus con 15 individuos, Pliocercus euryzonus con 13 individuos, Norops capito, Leptophis ahaetulla y Oxybelis fulgidus, con 12 individuos cada una, Basiliscus basiliscus, Spilotes pullatus y Norops humiles con 10 individuos cada una.

Cuadro 2. Composición y abundancia relativa de anfibios por familias y especies

\begin{tabular}{|c|c|c|c|}
\hline No. & Nombre científico & Abundancia relativa & Familia \\
\hline 1 & Bufo marinus & 3 & Bufonidae \\
\hline 2 & Bufo coccifer & 4 & Bufonidae \\
\hline 3 & Bufo haematiticus & 2 & Bufonidae \\
\hline 4 & Smilisca plaeota & 20 & Hylidae \\
\hline 5 & Smilisca baudinii & 5 & Hylidae \\
\hline 6 & Smilisca sordida & 2 & Hylidae \\
\hline 7 & Agalychnis callidryas & 10 & Hylidae \\
\hline 8 & Hyla ebraccata & 2 & Hylidae \\
\hline 9 & Agalychnis saltator & 2 & Hylidae \\
\hline 10 & Phyllomedusa lemur & 3 & Hylidae \\
\hline 11 & Agalychnis calcarifer & 2 & Hylidae \\
\hline 12 & Hyla loquax & 3 & Hylidae \\
\hline 13 & Ptychohyla hypomykter & 2 & Hylidae \\
\hline 14 & Leptodactylus melanonotus & 6 & Leptodactylidae \\
\hline 15 & Eleutherodactylus bransfordii & 4 & Leptodactylidae \\
\hline 16 & Eleutherodactylus ridens & 3 & Leptodatylidae \\
\hline 17 & Eleutherodactylus diastema & 3 & Leptodactylidae \\
\hline 18 & Eleutherodactylus fitzingeri & 2 & Leptodactylidae \\
\hline 19 & Eleutherodactylus biporcatus & 6 & Leptodactylidae \\
\hline 20 & Physalaemus pustulosus & 16 & Leptodactylidae \\
\hline 21 & Eleutherodactylus mimus & 7 & Leptodactylidae \\
\hline 22 & Eleutherodactylus noblei & 3 & Leptodactylidae \\
\hline 23 & Electherodactylus cerasinus & 8 & Leptodactylidae \\
\hline 24 & Centrolene ilex & 2 & Centrolenidae \\
\hline 25 & Cochranella granulosa & 3 & Centrolenidae \\
\hline 26 & Hyalinobatrachium pulveratur & 3 & Centrolenidae \\
\hline 27 & Centrolene prosoblepon & 2 & Centrołnidae \\
\hline 28 & Rana berlandieri & 2 & Ranidae \\
\hline 29 & Rana vaillanti & 5 & Ranidae \\
\hline 30 & Gymnopis multiplicata & 4 & Caeciliaidae \\
\hline 31 & Oedipina Sp & 6 & Plethodontidae \\
\hline
\end{tabular}

Fuente: Elaboración propia.

Las familias más representadas en anfibios fueron: Leptodactylidae con 10 especies y 58 individuos, Hylidae con 10 especies y 51 individuos y Centrolenidae con 4 especies y 10 individuos. Las familias menos representativas fueron: Bufonidae con 3 especies y 20 
individuos, Ranidae con 2 especies y 7 individuos, Caeciliaidae con 1 especie Gymnopis multiplicata y 4 individuos y Plethodontidae con 1 especie Oedipina Sp con 6 individuos.

De las especies de anfibios hay que destacar la presencia de Phyllomedusa lemur de la familia Hylidae que se encontró tres veces y no estaba reportado para el país. Hay que destacar la presencia del género Oedipina Sp, de la familia Plethodontidae que puede ser una especie nueva o un reporte más para el país o la zona.

Las especies más abundantes durante el estudio fueron: Smilisca plaeota con 20 individuos, siendo la especie más representativa, seguido de Physalaemus pustulosus con 16 individuos, Agalychnis callydrias con 10 individuos y Eleutherodactylus cerasinus con 8 individuos.

Cuadro 3. Abundancia relativa de reptiles por tipos de microhábitats

\begin{tabular}{|c|c|c|c|c|c|c|}
\hline \multirow[b]{2}{*}{ No. } & \multirow[b]{2}{*}{ ESPECIES } & \multicolumn{5}{|c|}{ Tipos de Microhábitats } \\
\hline & & Suelo/hojarasca & Fuste & Follaje/ramas & Riachuelos/rocas & $\begin{array}{l}\text { Abun. } \\
\text { relativa }\end{array}$ \\
\hline 1 & Norops humilis & 0 & 1 & 1 & 0 & 2 \\
\hline 2 & Norops cupreus & 0 & 1 & 1 & 0 & 2 \\
\hline 3 & Norops lemurinus & 1 & 1 & 1 & 0 & 3 \\
\hline 4 & Norops capito & 1 & 1 & 1 & 0 & 3 \\
\hline 5 & Norops biporcatus & 1 & 1 & 0 & 0 & 2 \\
\hline 6 & Sceloporus malachiticus & 1 & 0 & 0 & 1 & 2 \\
\hline 7 & Basiliscus plumifrons & 0 & 1 & 1 & 1 & 3 \\
\hline 8 & Basiliscus basiliscus & 1 & 1 & 0 & 1 & 3 \\
\hline 9 & Leptophis ahaetulla & 0 & 1 & 1 & 1 & 3 \\
\hline 10 & Imantodes cenchoa & 0 & 0 & 1 & 0 & 1 \\
\hline 11 & Drymobius margaritifer & 1 & 0 & 0 & 0 & 1 \\
\hline 12 & Lampropeltis triangulu & 1 & 0 & 0 & 0 & 1 \\
\hline 13 & Oxybelis fulgidus & 0 & 1 & 1 & 0 & 2 \\
\hline 14 & Ninia maculata & 1 & 0 & 1 & 0 & 2 \\
\hline 15 & Pliocercus euryzonus & 1 & 0 & 0 & 0 & 1 \\
\hline 16 & Rhadinaea decorata & 1 & 0 & 0 & 0 & 1 \\
\hline 17 & Spilotes pullatus & 0 & 0 & 1 & 0 & 1 \\
\hline 18 & Stenorrhina freminville & 1 & 0 & 1 & 0 & 2 \\
\hline 19 & Drymobius chloroticus & 0 & 0 & 0 & 1 & 1 \\
\hline 20 & Dryadophis dorsalis & 0 & 0 & 1 & 0 & 1 \\
\hline 21 & Hydromorphus concolor & 0 & 0 & 1 & 0 & 1 \\
\hline 22 & Oxyrhopus petola & 1 & 0 & 0 & 0 & 1 \\
\hline 23 & Oxybelis brevirostris & 0 & 0 & 1 & 1 & 2 \\
\hline 24 & Nothopsis rugosus & 1 & 0 & 0 & 1 & 2 \\
\hline 25 & Pseustes poecilonotus & 0 & 0 & 1 & 0 & 1 \\
\hline 26 & Sibon annulatus & 1 & 0 & 1 & 0 & 2 \\
\hline 27 & Atropoides nummifer & 1 & 0 & 0 & 0 & 1 \\
\hline 28 & Lachesis muta & 1 & 0 & 0 & 0 & 1 \\
\hline 29 & Agkistrodon bilineatus & 1 & 0 & 0 & 0 & 1 \\
\hline 30 & Bothriechis schlegelii & 0 & 1 & 1 & 0 & 2 \\
\hline 31 & Porthidium nasutum & 1 & 0 & 0 & 0 & 1 \\
\hline 32 & Celestus bivittatus & 1 & 0 & 0 & 0 & 1 \\
\hline 33 & Sphenomorphus cherriet & 1 & 0 & 0 & 0 & 1 \\
\hline 34 & Micrurus nigrocintus & 1 & 0 & 0 & 1 & 2 \\
\hline 35 & Boa constrictor & 1 & 1 & 1 & 0 & 3 \\
\hline $\mathrm{Abu}$ & dancia $(\mathrm{N})$ & 22 & 11 & 18 & 8 & 59 \\
\hline
\end{tabular}


De las frecuencias de individuos por microhábitat se obtuvieron los siguientes datos: la mayor frecuencia para el caso de reptiles fue observada en suelo/hojarasca $(\mathrm{N}=22)$, follaje/ ramas $(\mathrm{N}=18)$ y fuste $(\mathrm{N}=11)$, en menor frecuencia se observó en riachuelos/rocas $(\mathrm{N}=8)$ y se obtuvo un total de abundancia relativa de 59 en los cuatro microhábitats.

Se determinaron como especies comunes en el microhábitat suelo/hojarasca: con una frecuencia de tres, Norops lemurinus, Norops capito, Basiliscus basiliscus y Boa constrictor; con una frecuencia de dos, Norops biporcatus, Sceloporus malachiticus, Ninia maculata, Stenorrhina freminvillei, Nothopsis rugosus, Sibon annulatus y Micrurus nigrocintus; y con una frecuencia de uno, Drymobius margaritiferus, Lampropeltis triangulum, Pliocercus euryzonus, Rhadinaea decorata, Oxyrhopus petola, Atropoides nummifer, Lachesis muta, Agkistrodon bilineatus, Porthidium nasutum, Celestus bivittatus y Sphenomorphus cherriei. En el microhábitat fuste: con una frecuencia de tres, Norops lemurinus, Norops capito, Basiliscus plumifrons, Basiliscus basiliscus, Leptophis ahaetulla y Boa constrictor; con una frecuencia de dos Norops humilis, Norops cupreus, Oxybelis fulgidus, Oxybelis fulgidus y Bothriechis schlegelii. En el microhábitat follaje/ramas: con una frecuencia de tres, Norops lemurinus, Norops capito, Basiliscus plumifrons, Leptophis ahaetulla y Boa constrictor, con una frecuencia de dos, Norops humilis, Norops cupreus, Oxybelis fulgidus, Ninia maculata, Stenorrhina freminvillei, Oxybelis brevirostris, Sibon annulatus y Bothriechis schlegelii; y con una frecuencia de uno Imantodes cenchoa, Spilotes pullatus, Dryadophis dorsalis, Sibon dimidiatus y Pseustes poecilonotus. Y en el microhábitat riachuelos/rocas: con una frecuencia de tres, Basiliscus plumifrons, Basiliscus basiliscus y Leptophis ahaetulla; con una frecuencia de dos, Sceloporus malachiticus, Oxybelis brevirostris, Nothopsis rugosus y Micrurus nigrocintus; y con una frecuencia de uno, únicamente Drymobius chloroticus. 
Cuadro 4. Abundancia relativa de anfibios por tipos de microhábitats

\begin{tabular}{|c|c|c|c|c|c|c|}
\hline \multirow[b]{2}{*}{ No. } & \multirow[b]{2}{*}{ ESPECIES } & \multicolumn{5}{|c|}{ Tipos de Microhábitats } \\
\hline & & Suelo/hojarasca & Fuste & Follaje/ramas & Riachuelo/rocas & $\begin{array}{l}\text { Abun. } \\
\text { relativa }\end{array}$ \\
\hline 1 & Bufo marinus & 1 & 0 & 0 & 0 & 1 \\
\hline 2 & Bufo valliceps & 1 & 0 & 1 & 1 & 3 \\
\hline 3 & Bufo haematiticus & 1 & 0 & 0 & 1 & 2 \\
\hline 4 & Smilisca plaeota & 0 & 0 & 0 & 1 & 1 \\
\hline 5 & Smidsca baudinii & 0 & 1 & 0 & 1 & 2 \\
\hline 6 & Smilisca sordida & 0 & 0 & 0 & 1 & 1 \\
\hline 7 & Agalychnis callidryas & 0 & 0 & 1 & 1 & 2 \\
\hline 8 & Hyla ebraccata & 0 & 0 & 0 & 1 & 1 \\
\hline 9 & Agalychnis saltator & 0 & 0 & 1 & 1 & 2 \\
\hline 10 & Phyllomedusa lemur & 0 & 1 & 0 & 1 & 2 \\
\hline 11 & Agalychnis calcarifer & 0 & 0 & 1 & 1 & 2 \\
\hline 12 & Hyla loquax & 0 & 0 & 0 & 1 & 1 \\
\hline 13 & Ptychohyla hypomykter & 0 & 0 & 0 & 1 & 1 \\
\hline 14 & Leptodactylus melanonotus & 1 & 0 & 0 & 1 & 2 \\
\hline 15 & Eleutherodactylus bransfordix & 1 & 0 & 0 & 0 & 1 \\
\hline 16 & Eleutherodactylus ridens & 1 & 0 & 0 & 0 & 1 \\
\hline 17 & Eleutherodactylus diastema & 1 & 0 & 0 & 0 & 1 \\
\hline 18 & Eleutherodactylus fitzingeri & 1 & 0 & 0 & 0 & 1 \\
\hline 19 & Eleutherodactylus biporcatus & 1 & 0 & 0 & 0 & 1 \\
\hline 20 & Physalaemus pustulosus & 1 & 0 & 0 & 1 & 2 \\
\hline 21 & Eleutherodactylus mimus & 1 & 0 & 0 & 1 & 2 \\
\hline 22 & Eleutherodactylus noblei & 1 & 0 & 0 & 0 & 1 \\
\hline 23 & Electherodactylus cerasinus & 1 & 0 & 0 & 0 & 1 \\
\hline 24 & Centrolene ilex & 0 & 0 & 0 & 1 & 1 \\
\hline 25 & Cochranella granulosa & 0 & 0 & 1 & 1 & 2 \\
\hline 26 & Hyalinobatrachium pulveratu & 0 & 0 & 1 & 1 & 2 \\
\hline 27 & Centrolene prosoblepon & 0 & 0 & 0 & 1 & 1 \\
\hline 28 & Rana berlandieri & 1 & 0 & 0 & 1 & 2 \\
\hline 29 & Rana vaillanti & 0 & 0 & 0 & 1 & 1 \\
\hline 30 & Gymnopis multiplicata & 1 & 0 & 0 & 0 & 1 \\
\hline 31 & Oedipina $S p$ & 1 & 0 & 0 & 0 & 1 \\
\hline Abun & dancia $(\mathrm{N})$ & 16 & 2 & 6 & 21 & 45 \\
\hline
\end{tabular}

De las frecuencias de individuos por microhábitat, la mayor frecuencia para el caso de anfibios fue observada en los microhabitats riachuelos/rocas ( $\mathrm{N}=21)$ y suelo/hojarasca $(\mathrm{N}=16)$, mientras que en menor frecuencia fue observada en los microhábitats fuste $(\mathrm{N}=6)$ y follaje/ramas $(\mathrm{N}=2)$, presentando un total de abundancia relativa de 45 en los cuatro tipos de microhábitats.

Se determinaron como especies comunes en el microhábitat suelo/hojarasca: con una frecuencia de tres, únicamente Bufo valliceps; con una frecuencia de dos, Bufo haemati ticus,Leptodactylus melanonotus, Physalaemus pustulosus, Eleutherodactylus mimus y Rana berlandieri; y con una frecuencia de uno, Bufo marinus, Eleutherodactylus bransfordii, Eleutherodactylus ridens, Eleutherodactylus diastema, Eleutherodactylus fitzingeri, Eleutherodactylus biporcatus, Eleutherodactylus noblei, Electherodactylus cerasinus, Gymnopis multiplicata y Oedipina Sp. En el microhábitat fuste: con una frecuencia de dos, únicamente dos especies, Smilisca baudinii y Phyllomedusa lemur. En el microhábitat follaje/ 
ramas: con una frecuencia de tres, únicamente Bufo valliceps; con una frecuencia de dos Agalychnis callidryas, Agalychnis saltator, Agalychnis calcarifer, Cochranella granulosa y Hyalinobatrachium pulveratum. Y en el microhábitat riachuelos/rocas: con una frecuencia de tres, únicamente Bufo valliceps; con una frecuencia de dos Bufo haematiticus, Smilisca baudinii, Agalychnis callidryas, Agalychnis saltator, Phyllomedusa lemur, Agalychnis calcarifer, Leptodactylus melanonotus, Physalaemus pustulosus, Eleutherodactylus mimus, Cochranella granulosa, Hyalinobatrachium pulveratum y Rana berlandieri; y con una frecuencia de uno Smilisca plaeota, Smilisca sordida, Hyla ebraccata, Hyla loquax, Ptychohyla hypomykter, Centrolene ilex, Centrolene prosoblepon y Rana vaillanti.

Cuadro 5. Índices de diversidad de reptiles por tipos de microhábitats

\begin{tabular}{|c|c|c|c|c|}
\hline \multirow{2}{*}{ Índices } & & \multicolumn{3}{|c|}{ Tipos de microhábitats } \\
\cline { 2 - 5 } & Suelo/hojarasca & Fuste & Follaje/ramas & Riachuelo/rocas \\
\hline Riqueza & & & & \\
$\mathrm{S}$ & 22 & 11 & 18 & 8 \\
\hline Diversidad & 0.16 & 0.23 & 0.15 & 0.21 \\
$\lambda$ & 2.40 & 1.80 & 2.74 & 1.74 \\
H' & & & & \\
& & & & 0.65 \\
Equitatividad & 0.77 & 0.81 & 0.72 & 0.61 \\
E1 & 0.62 & 0.82 & 0.60 & \\
E5 & & & & \\
\hline
\end{tabular}

El cálculo de los índices de diversidad demuestra que los microhabitats más diversos para el caso de reptiles son follaje/ramas $\left(H^{\prime}=2.74, \lambda=0.15\right)$ y suelo/hojarasca $\left(H^{\prime}=2.40\right.$, $\lambda=0.16)$, y la menor diversidad se encuentra en fuste $\left(H^{\prime}=1.80, \lambda=0.23\right)$ y riachuelos/rocas $\left(H^{\prime}=1.74, \lambda=0.21\right)$. Según lo expresado por los índices de E1 y E5 las especies de reptiles se distribuyeron más homogéneamente en los microhábitats fuste $(\mathrm{E} 1=0.81$, E5=0.82), suelo/ hojarasca $(\mathrm{E} 1=0.77, \mathrm{E} 5=0.62)$ y follaje/ramas $(\mathrm{E} 1=0.72, \mathrm{E} 5=0.60)$, presentando menor equitatividad en riachuelos/rocas $(\mathrm{E} 1=0.65, \mathrm{E} 5=0.61)$. La riqueza de especies fue mayor en los microhabitats suelo/hojarasca (22), follaje/ramas (18) y fuste (11), presentando menor riqueza el microhábitat riachuelos/rocas, con (8). 
Cuadro 6. Índices de diversidad de anfibios por tipos de microhábitats

\begin{tabular}{c|c|c|c|c|}
\hline \multirow{2}{*}{ Índices } & & \multicolumn{3}{|c|}{ Tipos de microhábitats } \\
\cline { 2 - 5 } & Suelo/hojarasca. & Fuste & Follaje/ramas & Riachuelo/rocas \\
\hline $\begin{array}{c}\text { Riqueza } \\
\text { S }\end{array}$ & 16 & 2 & 6 & 21 \\
& & & & \\
\hline $\begin{array}{c}\text { Diversidad } \\
\lambda\end{array}$ & 0.40 & 0.30 & 0.19 & 0.15 \\
H' & 2.72 & 0.95 & 1.72 & 2.38 \\
& & & & \\
\hline $\begin{array}{c}\text { ¿quitatividad } \\
\text { E1 }\end{array}$ & 0.70 & 0.85 & 0.77 & 0.76 \\
E5 & 0.52 & 1.28 & 0.78 & 0.56 \\
\hline
\end{tabular}

El calculo de los índices de diversidad demuestra que los microhábitats más diversos para el caso de anfibios son suelo/hojarasca $\left(H^{\prime}=2.72, \lambda=0.40\right)$, riachuelos/rocas $\left(H^{\prime}=238, \lambda=0.15\right)$ y follaje/ramas $\left(H^{\prime}=172, \lambda=0.19\right)$ y la menor diversidad se presenta en fuste $\left(H^{\prime}=0.95, \lambda=0.30\right)$. Según lo expresado por los índices de E1 y E5 las especies de anfibios se distribuyeron más homogéneamente en los microhábitats fuste $(\mathrm{E} 1=0.85$, $\mathrm{E} 5=1.28)$, follaje/ramas $(\mathrm{E} 1=0.77$, $\mathrm{E} 5=0.78)$ y riachuelos/rocas $(\mathrm{E} 1=0.76, \mathrm{E} 5=0.56)$, presentando menor equitatividad en suelo/hojarasca $(\mathrm{E} 1=0.70, \mathrm{E} 5=0.52)$. La riqueza de especies fue mayor en los microhábitats riachuelos/rocas (21), suelo/hojarasca (16) y follaje/ramas (6), presentando menor riqueza el microhábitat fuste con (2).

\section{Índices generales de Shannon y Simpson para el área:}

Sobre la base de los índices calculados de Shannon y Simpson por tipos de microhábitats, se obtuvo un índice general para el área que para el caso de reptiles fue: $\mathrm{H}^{\prime}=1.50$ y $\lambda=0.25$ y para el caso de anfibios fue: $\mathrm{H}^{\prime}=0.60 \mathrm{y} \lambda=0.28$

\section{Comparación de la composición de la herpetofauna entre tipos de microhábitats:}

Los microhábitats que obtuvieron él más alto valor de asociación en reptiles fueron: fuste y riachuelos/rocas por tener la mayoría de las especies de reptiles más comunes. Los microhábitats menos parecidos fueron: suelo/hojarasca y follaje/ramas por tener la mayoría de las especies de reptiles menos comunes.

En el taxa anfibios los microhábitats que obtuvieron el más alto valor de asociación fueron: follaje/ramas y fuste por tener la mayoría de las especies de anfibios más comunes. Los microhábitats menos parecidos fueron: riachuelos/rocas y suelo/hojarasca por tener la mayoría de las especies de anfibios menos comunes. 


\section{Conclusiones}

Las familias más representativas de reptiles fueron Colubridae, Iguanidae y Viperidae, y de anfibios fueron Leptodactylidae, Hylidae y Centrolenidae, todas por presentar el mayor número de especies.

Las especies más comunes de reptiles fueron: Micrurus nigrocintus, Sceloporus malachiticus, Pliocercus euryzonus, Norops capito, Leptophis ahaetulla, Oxybelis fulgidus, Basiliscus basiliscus, Spilotes pullatus y Norops humiles. Las especies más comunes deanfibios fueron:Smilisca plaeota (especiemuyrepresentativa del área), Physalaemus pustulosus, Agalychnis callydrias y Eleutherodactylus cerasinus.

Es muy importante el hallazgo de dos géneros y dos especies. Phyllomedusa lemur de la familia Hylidae, encontrada tres veces, podría ser un reporte nuevo para Nicaragua. El género Oedipina $\mathbf{S p}$, de la familia Plethodontidae podría ser un reporte nuevo también para el país y la zona.

El área de estudio fue heterogénea en su composición de anfibios y reptiles entre microhábitats. Los cuatro microhábitats fueron heterogéneos por presentar especies asociadas a ellos.

\section{Recomendaciones}

5.1. Realizar estudios sobre heterogeneidad ecológica y fisiográfica de la comunidad herpetofaunística para determinar el uso y preferencias de hábitats por parte de las especies en el área de estudio.

5.2. Incrementar el monitoreo sobre la diversidad de la herpetofauna de manera sistemática a largo plazo en el área protegida para obtener mayor información sobre su ecología, distribución y abundancia sobre todas aquellas especies que son consideradas o representan reportes nuevos para el país o la zona.

5.3. Evaluar costos y beneficios ambientales del aprovechamiento de las fuentes hídricas naturales para diferentes actividades por parte de las haciendas aledañas al área protegida, valorando el impacto ecológico que ocasiona el corte de caudal o la ausencia de estas fuentes sobre la disminución de nichos ecológicos para anfibios y reptiles en los sitios de galerías.

5.4. Concienciar a productores y localidades cercanas al área protegida sobre la importancia y beneficios de estos animales por ser atractivos turísticos y controladores biológicos de diferentes plagas en la naturaleza. 


\section{Agradecimientos}

Agradecemos la colaboración del MSc. Gustavo Adolfo Ruiz con el apoyo en la identificación de especies. También queremos agradecer a la Dirección de Investigación de la Universidad Centroamericana (UCA) por el apoyo económico brindado a través del programa FIUCA. Al Lic. Gabriel Bolaños por su colaboración y acompañamiento en el campo. A Giovanna Valenti por su colaboración. Al personal de seguridad de Santa Maura y la Estación Biológica de la UCA, como también a Guarda Parques del Goviado por su asistencia y colaboración logística en el campo. Agradecemos especialmente al Padre Adolfo López SJ por su apoyo incondicional, a la Lic. Vera Amanda Solís, decana de la Facultad de Ciencia, Tecnología y Ambiente de la UCA, por la gestión realizada. A la Lic. Janina Urcuyo por sus recomendaciones y apoyo incondicional de siempre. Asimismo, se les agradece a todas las personas que colaboraron con nuestra investigación.

\section{Referencias bibliográficas}

BACH, O. (1998). Diversidad, abundancia y distribución espacial de la herpotofauna en tres líneas bananeras bajo diferentes manejos agrícolas en la Zona Atlántica de Costa Rica. Tesis de maestría. Universidad Nacional de Costa Rica: Heredia.

HEYER, W. R.; DONNELLY, M.A.; MCDIARMID, R.W.; HAYER, L.A.C. \& FUSTER, M.S. (1994). Measuring and Monitoring Biological Diversity: Standard Methods for Amphibians. Smithsonian Institution Press: Washington and London.

INCER, J. (1998). Geografía dinámica de Nicaragua. Editorial Hispamer: Managua.

KÖLHER, G. (1998). The Amphibians and Reptiles of Nicaragua. A.M: Frankfurt, Germany.

KÖLHER, G. (2001). Anfibios y reptiles de Nicaragua. Herpeton: Offlenbach, Alemania.

KREBS, J. C. (1989). Ecología: Estudio de la distribución y la abundancia. Harla, S. A.: México, D. F. $2^{\text {a }}$ edición.

LUDWIG, J. \& REYNOLDS, J. (1988). Statistical Ecology: A Primer on Methods and Computing. A Wiley-Interscience, Publication, John Wiley \& Sons, Inc.: United States of America.

MAGURRAN, A. (1988). Ecological Diversity and Its Measurement. Princeton University Press: Princeton, New Jersey.

RUÍZ, G. A. (1996). Claves preliminares para reconocer a los reptiles de Nicaragua. Cedaprode (Centro de Derecho Ambiental y Promoción para el Desarrollo): Managua.

RUÍZ, G. A. \& Buitrago. F. (2003). Guía ilustrada de la herpetofauna de Nicaragua. ARAUCARIA-MARENA-AECI: Managua. $1^{\text {a }}$ Edición. 